\title{
A flux-mnemonic permanent magnet brushless motor for electric vehicles
}

\author{
Chuang $\mathrm{Yu}^{\mathrm{a})}$ and K. T. Chau \\ Department of Electrical and Electronic Engineering, University of Hong Kong, Hong Kong, China
}

Xinhua Liu and J. Z. Jiang

School of Electromechanical Engineering and Automation, Shanghai University, Shanghai, 20072 China

(Presented on 8 November 2007; received 6 September 2007; accepted 11 October 2007; published online 18 January 2008)

\begin{abstract}
In this paper, a new permanent magnet (PM) brushless motor is proposed for electric vehicles. The key is to incorporate the concept of memory motors, namely, the online tunable flux-mnemonic PMs, into the stator doubly fed doubly salient PM motor, hence achieving effective air-gap flux control. By further employing the outer-rotor and double-layer-stator topology, the proposed motor takes the definite advantages of compact structure, low armature reaction, and direct-drive capability. Increasingly, this motor can offer the unique features of pole dropping and pole reversing. Finite element analysis and, hence, computer simulation are given to verify the validity of the proposed motor. @ 2008 American Institute of Physics. [DOI: 10.1063/1.2830553]
\end{abstract}

\section{INTRODUCTION}

With ever increasing concerns on environment protection and energy conservation, electric vehicles (EVs) have been identified as the most viable zero-emission vehicles. ${ }^{1}$ Among different EV motors, the permanent magnet brushless (PMBL) motor is most attractive since it inherently offers the merits of high efficiency and high power density. ${ }^{2}$ However, because of uncontrollable air-gap flux, the PMBL motor cannot offer the desired constant-power operating range for EV cruising. In recent years, some attempts have been made to solve this problem. Based on the doubly salient PM (DSPM) motor, the PMs are replaced by dc field windings so that the resulting stator doubly fed doubly salient (SDF-DS) motor can control the air-gap flux. ${ }^{3}$ However, it significantly degrades the power density and efficiency. By incorporating PMs and air bridges into the SDF-DS motor, the resulting SDF-DSPM motor can retain the desired air-gap flux control while improving the power density and efficiency. ${ }^{4}$ Nevertheless, the use of dc field current inevitably causes additional power loss. With the advent of the memory motors, ${ }^{5}$ the magnetization of PMs becomes online tunable. However, it suffers from a complicated control of armature current for PM magnetization and the possibility of accidental demagnetization due to armature reaction especially during high-rate regenerative braking.

The purpose of this paper is to incorporate the concept of online tunable flux-mnemonic PMs into the SDF-DSPM motor in such a way that the resulting flux-mnemonic PMBL motor can offer effective and efficient air-gap flux control. The high effectiveness is due to its direct magnetization of PMs by field windings, while the high efficiency is due to the use of a temporary current pulse for PM magnetization.

\section{DESIGN}

The configuration of the proposed flux-mnemonic PMBL motor is shown in Fig. 1, which adopts a five-phase outer-rotor double-layer-stator topology. The use of five phases rather than three phases is to enhance the torque smoothness, which is desirable for vehicular operation. The use of outer rotor rather than inner rotor is to allow for direct in-wheel driving, which is highly desirable for modern EVs. Also, since the outer rotor is simply composed of salient poles with no PMs or windings, it is very robust and suitable for vehicular operation. In the stator, the armature windings are located in the outer layer, while both the PMs and field windings are located in the inner layer, hence achieving a compact structure. Moreover, since the armature windings and the PMs are located in different layers, and the armature adopts fractional-slot windings with the coil span equal to the slot pitch, the PMs can be immune from accidental demagnetization by armature reaction.

Similar to existing memory motors, the PM material used in the proposed motor is an aluminum nickel cobalt (AlNiCo) alloy. Its demagnetization curve can offer a high remanent flux density $B_{r}$ to enable high air-gap flux density

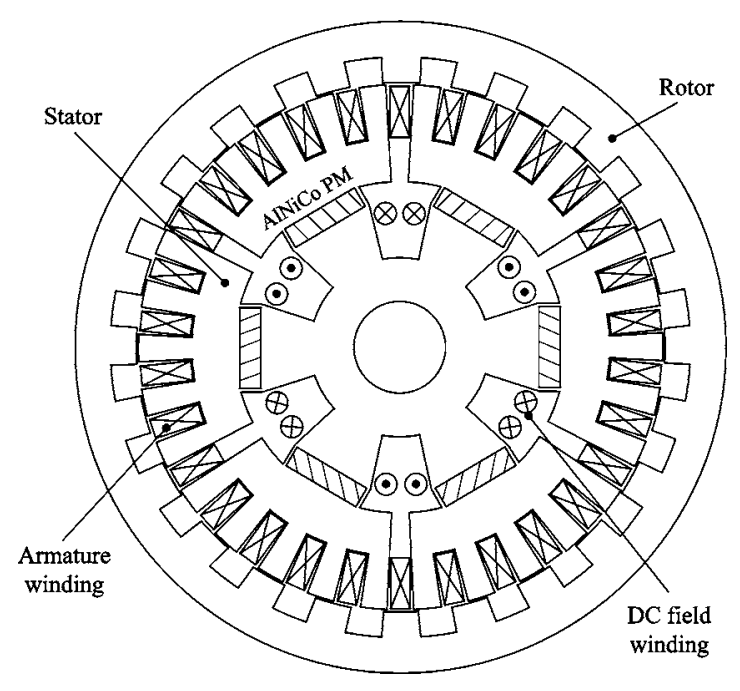

FIG. 1. Proposed motor configuration.

${ }^{a)}$ Electronic mail: chuangyu@eee.hku.hk. 


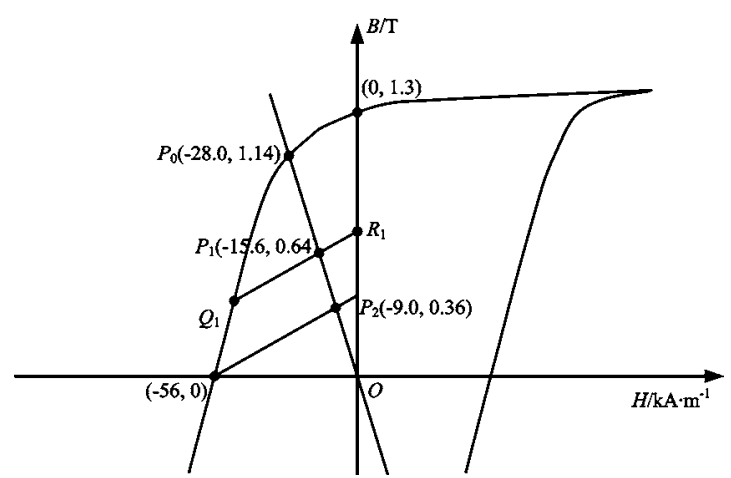

FIG. 2. Typical magnetization curve of PMs.



FIG. 4. Air-gap flux density distributions at different PM magnetizations.

and a relatively low coercive force $H_{c}$ to enable online magnetization. Different from existing memory motors, the PM magnetization level of the proposed motor is tuned by applying a current pulse to the field windings. Thus, it does not need to control the $d$-axis armature current, which is very complicated and may even conflict with the motor control strategy.

As depicted in Fig. 2, the level of full magnetization is denoted by the operating point $P_{0}$, which is the interaction of the demagnetization curve and the load line. In order to lower the PM magnetization level, a negative current pulse is applied so that the operating point shifts from $P_{0}$ to $Q_{1}$. After

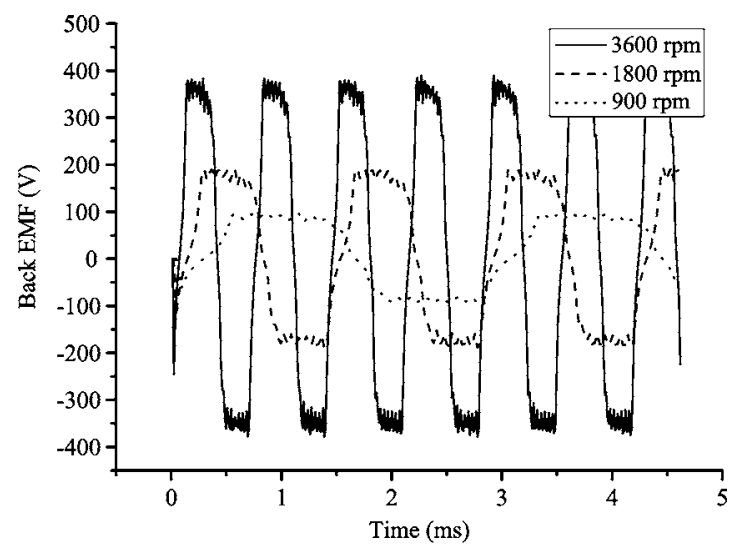

(a)

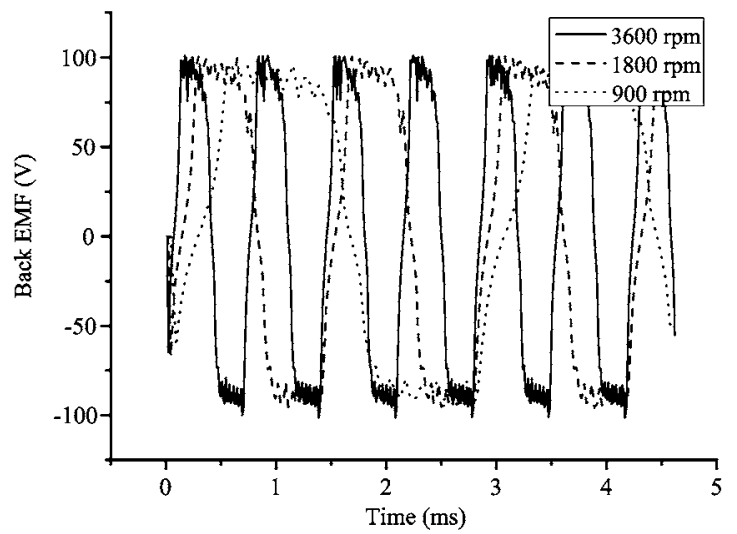

(b)

FIG. 5. Back EMF waveforms at different speeds. (a) Without flux control. (b) With flux control.
FIG. 3. Magnetic field distributions. (a) Full magnetization and no load. (b) Weak magnetization and no load. (c) No magnetization and full load. (d) Full magnetization and full load. (b)

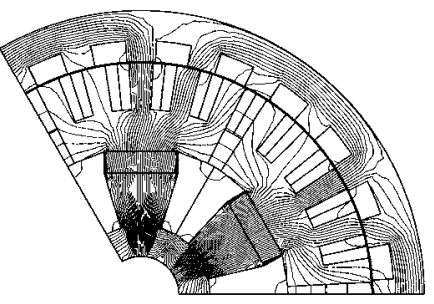

(d) 


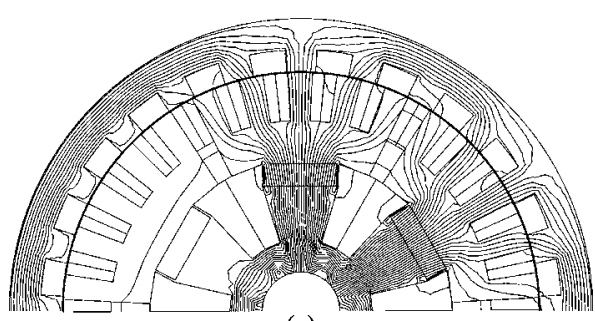

(a)

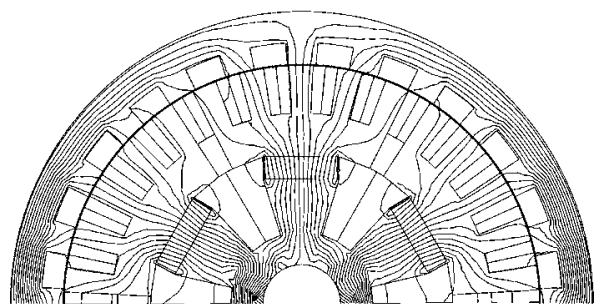

(b)

FIG. 6. Magnetic field distributions. (a) Four PM poles using pole dropping (b) Two PM poles using pole reversing.

this current pulse, it moves along the recoil line $Q_{1} R_{1}$ and then settles at $P_{1}$. Similarly, in order to raise the PM magnetization level, a positive current pulse is applied so that the operating point shifts back to $P_{0}$. Therefore, by adjusting the magnitude and polarity of the current pulse, the PM magnetization level and, hence, the air-gap flux density can be flexibly controlled.

\section{ANALYSIS}

The design data of the proposed motor is listed in Table I. By using finite element analysis, the magnetic field distributions under different PM magnetization levels and different loads are shown in Fig. 3. Figures 3(a) and 3(b) illustrate that the magnetic field strength changes in accordance with the PM magnetization levels, while Figs. 3(c) and 3(d) depict that the armature field concentrates on the outer layer of the stator, thus avoiding accidental demagnetization of the PMs.

The air-gap flux density distributions at different PM magnetization levels under no load are shown in Fig. 4. It can be found that the air-gap flux density can be effectively controlled for over four times. When the motor runs at $900 \mathrm{rpm}$ (rated speed), $1800 \mathrm{rpm}$, and $3600 \mathrm{rpm}$, the corresponding back electromotive force (EMF) waveforms with and without control are shown in Fig. 5. It confirms that by online tuning the PM magnetization levels, the back EMF can be maintained constant; hence, constant-power operation can be performed up to four times base speed.

The proposed motor exhibits two unique features, which are absent in literature. By totally demagnetizing or reversely magnetizing two of the six PM pieces, the number of PM poles becomes 4 (so-called pole dropping) or 2 (so-called pole reversing), respectively, as illustrated in Fig. 6. The corresponding effects at the rated speed are shown in Fig. 7,

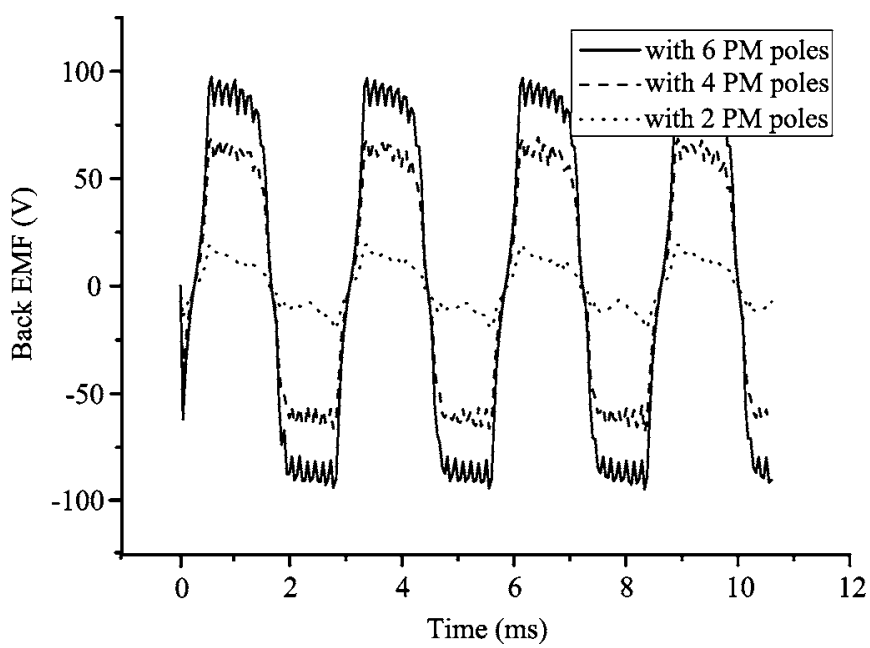

FIG. 7. Back EMF waveforms at rated speed under different numbers of PM poles.

which shows that the back EMF is reduced by $29.3 \%$ by using pole dropping and $84.7 \%$ by using pole-reversing. Hence, the back EMF can be maintained constant when using pole dropping at $1274 \mathrm{rpm}$ or using pole reversing at $5914 \mathrm{rpm}$. Nevertheless, in order to maintain the back EMF constant at other speeds, the control of magnetization levels of the PMs is still necessary.

\section{CONCLUSION}

A flux-mnemonic PMBL motor has been proposed for EVs. It not only inherits the advantages of effective flux control, high efficiency, and high power density from SDFDSPM motors, but also overcomes the problems of accidental demagnetization and complex control in memory motors. By simply applying temporary current pulses to the field winding, the PM magnetization levels and, hence, air-gap flux density can be effectively and efficiently controlled, leading to a constant-power operation up to four times base speed. Finally, this motor offers the features of pole dropping and pole reversing, which are meaningful for future development.

\section{ACKNOWLEDGMENTS}

This work is funded by a grant (HKU 7105/07E) of the Research Grants Council, Hong Kong Special Administrative Region, China.

${ }^{1}$ K. T. Chau and C. C. Chan, Proc. IEEE 95, 821 (2007).

${ }^{2}$ K. T. Chau, W. Cui, J. Z. Jiang, and Z. Wang, J. Appl. Phys. 99, 08 R322 (2006).

${ }^{3}$ M. Cheng, Y. Fan, and K. T. Chau, J. Appl. Phys. 97, 10Q508 (2005).

${ }^{4}$ K. T. Chau, Y. B. Li, J. Z. Jiang, and C. Liu, IEEE Trans. Magn. 42, 3470 (2006).

${ }^{5}$ V. Ostovic, IEEE Ind. Appl. Mag. 99, 52 (2003). 